\author{
Ljiljana S. Aćimović* \\ Universität in Banja Luka \\ Philologische Fakultät \\ Lehrstuhl für Germanistik
}

\title{
NARRATOLOGISCHE ANALYSE DER DARSTELLUNG DER KRANKHEIT IN DEN WERKEN DER ALTE KÖNIG IN SEINEM EXIL UND IN ZEITEN DES ABNEHMENDEN LICHTS
}

\author{
Original scientific paper \\ UDC 821.112.2(436) -312.6.09 Geiger A. \\ 821.112.2-321.6.09 Ruge E. \\ 616:82 \\ https://doi.org/10.18485/kkonline.2021.12.12.1
}

In diesem Beitrag wird untersucht, wie zwei deutschsprachige Autoren das Thema Krankheit darstellen. Beide Texte wurden gleichzeitig veröffentlicht, im Jahr 2011, und beide Werke weisen autobiografische Züge auf. Arno Geiger schreibt in seinem autobiografischen Text Der alte König in seinem Exil über seinen Vater August, der an Alzheimer erkrankt ist und an dessen Betreuung, Versorgung und Pflege sich alle vier Kinder, die getrennt lebende Ehefrau und im Haus lebende Pflegerinnen intensiv beteiligen. Demenz ist nur eine der Krankheiten, an der die Mitglieder der Familie Powileit/Umnitzer in Eugen Ruges Roman In Zeiten des abnehmenden Lichts leiden. Symptome der Demenzerkrankung sind bei Wilhelm Powileit und seinem Stiefsohn Kurt Umnitzer teilweise unterschiedlich und lassen sich mit denen in Geigers Text vergleichen. Ruges Roman ist auch stark autobiografisch geprägt. Er selbst wird durch die zentrale Roman- und Reflektorfigur Alexander Umnitzer vertreten. Der Roman beginnt und endet im Jahre 2001, als bei Alexander eine anscheinend unheilbare Krebserkrankung, das NonHodgkin-Lymphom, diagnostiziert wird. Er begibt sich nach der Diagnose auf eine Mexikoreise, nachdem er seinen schwer dementen Vater Kurt hilflos im Haus zurücklässt. Innere Ruhe findet er erst in einem kleinen Ort am Pazifik und diese Ruhe ermöglicht es ihm, über sein Leben, seine Krankheit und den Tod nachzudenken. Die ausgewählten Texte werden nach Kriterien der Narratologie auf der Diskursebene analysiert, wobei die Begriffe: Zeit, Modus und Stimme innerhalb der Textanalyse erläutert und analysiert werden. Im Schlussteil werden Gemeinsamkeiten und Unterschiede der analysierten Texte diskutiert.

Schlüsselwörter: Krankheit, Alzheimer, Krebs, Demenz, Alkoholismus

\section{Einleitung}

Krankheit als Phänomen und Bestandteil des Lebens weist eine lange Tradition der literarischen Bearbeitung auf. In der deutschsprachigen Literatur findet man eine sehr große Anzahl literarischer Texte, in denen sich die Autoren in verschiedener Form mit dem Thema Krankheit beschäftigen. An dieser Stelle erwähnen wir nur einige der bekanntesten Beispiele: Der arme Heinrich Hartmanns von Aue, Sebastian Brants Das

\footnotetext{
* Filološki fakultet, Bulevar vojvode Petra Bojovića 1A, 78000 Banja Luka, Republika Srpska, Bosna i Hercegovina; e-mail: ljiljana.acimovic@flf.unibl.org
} 
Narrenschiff, E. T. A. Hoffmanns Der Sandmann, Jeremias Gotthelfs Die schwarze Spinne, Thomas Manns Der Tod in Venedig. Heutzutage behandeln zeitgenössische Schriftsteller mit großem Interesse Krankheiten wie Krebs, Alzheimer, Demenz, verschiedene Abhängigkeiten oder psychische Störungen. In diesem Beitrag wird untersucht, auf welche Weise zwei zeitgenössische deutschsprachige Autoren das Thema Krankheit darstellen. Als Grundlage für die Untersuchung dienen Arno Geigers Buch Der alte König in seinem Exil und Eugen Ruges Roman In Zeiten des abnehmenden Lichts. Beide Texte wurden im gleichen Jahr veröffentlicht, 2011, und beide Werke weisen autobiografische Züge auf.

Arno Geiger (1968) erzählt in seinem im Carl Hanser Verlag veröffentlichten Buch über seinen an Alzheimer erkrankten Vater August. Der alte König in seinem Exil wurde für den Preis der Leipziger Buchmesse 2011 nominiert. ${ }^{1}$

Demenz ist nur eine der Krankheiten, die Eugen Ruge (1954) in seinem Roman thematisiert. Neben Demenz rücken bei ihm auch Alexanders Krebserkrankung und Irinas Alkoholismus in den Vordergrund. Der stark autobiografisch geprägte Roman wurde mehrfach ausgezeichnet: 2009 mit dem Alfred-Döblin-Preis, 2011 mit dem Aspekte-Literaturpreis und mit dem Deutschen Buchpreis. ${ }^{2}$ Das Buch wurde 2017 verfilmt.

Da der Gegenstand dieses Beitrags das Thema Krankheit ist, soll im Rahmen der Arbeit analysiert werden, wie Krankheit in den ausgewählten Werken dargestellt wird? Als theoretische Grundlage für die Analyse dient vor allem Matías Martínez' und Michael Scheffels Buch Einführung in die Erzähltheorie (2016), sowie folgende literaturtheoretische Abhandlungen: Jürgen H. Petersens Erzäh/systeme. Eine Poetik epischer Texte (1993), Eberhard Lämmerts Bauformen des Erzählens (1955) und Jochen Vogts Aspekte erzählender Prosa. Eine Einführung in Erzähltechnik und Romantheorie (1998). Die wichtigsten Fragen, die uns interessieren, sind: Wie wird die Krankheit/der Erkrankte im Text dargestellt? Wie wird die Erzählung präsentiert? Welche erzähltheoretische Strategien benutzt der Autor in der Darstellung der Krankheit(en)? Wir hoffen, mithilfe narratologischer Untersuchung auf der

\footnotetext{
${ }^{1}$ Das Buch wurde 2012 in Serbien von Nikola B. Cvetković übersetzt und unter dem Titel Kao stari kralj u izgnanstvu im Laguna Verlag veröffentlicht. Im gleichen Jahr erschien in Kroatien im Verlag Mozaik knjiga die Übersetzung des Buches von Branka Grubić unter dem Titel Priča o starom kralju koji nije mogao pronaći svoj dom.

2 Ruges Roman wurde 2014 in Kroatien unter dem Titel Kad svjetlost nestaje übersetzt und im OceanMore Verlag veröffentlicht. Die Übersetzerin ist Helen Sinković. In Bosnien-Herzegowina wurde das Buch im gleichen Jahr übersetzt und erschien im Buybook Verlag unter dem Titel $U$ vremenima zamirućeg svjetla. Mira Đorđević, Professorin der deutschsprachigen Literatur, übesetzte diese Ausgabe.
} 
Diskursebene zeigen zu können, auf welche Weise Arno Geiger und Eugen Ruge das Thema Krankheit problematisieren.

\section{Erzähltheoretische Grundlagen}

Mit der Frage „Wie lässt sich der Gegenstand der Erzähltheorie aus literaturwissenschaftlicher Sicht bestimmen?" (2016: 11) beginnen Martínez und Scheffel ihr Buch Einführung in die Erzäh/theorie, das heute zum unumgänglichen Handbuch der literaturwissenschaftlichen Analyse avancierte. Aus ihrer Sicht ist eine der wichtigsten Fragen der Erzähltheorie die Bestimmung "des fundamentalen Gegensatzes zwischen dem ,Wie' und dem ,Was' von Erzählungen" (2016: 22), wobei sie "zwischen dem erzählerischen Medium mitsamt den jeweils verwendeten Verfahren der Präsentation einerseits und dem Erzählen (die Geschichte, die erzählte Welt) andererseits" unterscheiden (2016: 22).

"Als ,Erzählen", so Martínez und Scheffel, „bezeichnet man eine Art von mündlicher oder schriftlicher Rede, in der jemand jemandem etwas Besonderes mitteilt" (2016: 11), und die Rede, von der die Literaturtheoretiker reden, heißt "offenbar eine ,Erzählung', wenn diese Rede einen ihr zeitlich vorausliegenden Vorgang vergegenwärtigt, der als ,Geschehnis' oder ,Begebenheit' bestimmt werden kann" (2016: 12).

Die Autoren betonen, dass „die Unterscheidung zwischen dem ,Was' und dem ,Wie' eines Erzähltextes häufig mit dem im Russischen Formalismus formulierten Gegensatz von ,fabula' und ,sjužet" in Zusammenhang gebracht" wird (2016: 25). Um diese Begriffe näher zu bestimmen, definieren sie die angeführten Termini von Boris Tomaševskij, Tzvetan Todorov und Émile Benveniste und schlussfolgern, dass "die genannten Begriffspaare einander ähneln, sind aber nicht bedeutungsgleich" (2016: 25). Sie wollen die Vermischung zwischen diesen Begriffen vermeiden und die genannten Termini berücksichtigend unterscheiden sie "die erzäh/te Welt (oder Diegese) von dem engeren Begriff der Handlung, der sich nur auf die Gesamtheit der handlungsfunktionalen Elemente der dargestellten Welt bezieht. Die andere Seite der Opposition, also die Art und Weise der Vermittlung der erzählten Welt, bezeichnen wir als Darstellung" (2016: 26).

Der französische Literaturtheoretiker Gérard Genette hat anstelle der Opposition von fabula/histoire vs. sjužet/discours eine Dreiteilung vorgeschlagen. $\mathrm{Er}$ unterscheidet drei Bedeutungen des Begriffs Erzählung. In der ersten Bedeutung des Wortes, schreibt Genette, „bezeichnet Erzählung die narrative Aussage, den 
mündlichen oder schriftlichen Diskurs [discours], der von einem Ereignis oder einer Reihe von Ereignissen berichtet" (2010: 11). In der zweiten Bedeutung ist Erzählung "die Abfolge der realen oder fiktiven Ereignisse, die den Gegenstand dieser Rede ausmachen und ihre unterschiedlichen Beziehungen zueinander - solche des Zusammenhangs, des Gegensatzes, der Wiederholung usw." (2010: 11). Die dritte Bedeutung des Wortes, nach Genette vielleicht die älteste, bezieht sich „auf den Akt der Narration selber", der darin besteht, "dass jemand etwas erzählt" (2010: 11). Um die Verwechslung dieser drei Bedeutungen zu vermeiden, schlägt er für die erste Bedeutung des Wortes den Begriff Erzählung im eigentlichen Sinne vor, für die zweite Bedeutung Geschichte und für die dritte Bedeutung Narration (2010: 12) Der Gegenstand der theoretischen Untersuchung ist nach ihm die Erzählung in der ersten Bedeutung des Wortes, d. h. die Erzählung in dem engeren Sinn (2010: 12).

Martínez und Scheffel meinen, dass "Genettes Dreiteilung in Form eines gleichberechtigten Nebeneinander von ,Geschichte', ,Erzählung' und ,Narration' jedoch nicht befriedigend" ist (2016: 26-27) und schlagen vor, "Genettes Dreiteilung als Anregung für eine Binnendifferenzierung zu nehmen, welche die Seite der Darstellung betrifft. Im Rahmen der grundsätzlichen Opposition von Welt oder Handlung vs. Darstellung unterscheiden wir deshalb, innerhalb des Feldes der Darstellung, zwischen ,Erzählung' und ,Erzählen"' (2016: 27). In diesem Sinne differenzieren die Autoren innerhalb der Erzählebene, in Anlehnung an Genettes Theorie, "zwischen der Erzählung (oder auch dem ,Text der Geschichte', verstanden als der schriftliche oder mündliche Diskurs, der von einem Geschehen erzählt) und dem Erzählen (verstanden als der Akt, der diesen Diskurs hervorbringt)" (2016: 32), wobei sie ihr Beschreibungsmodell nach drei Kategorien ordnen: Zeit, Modus und Stimme (2016: 32). Im Rahmen dieser Arbeit werden wir diese drei Kategorien: Zeit, Modus und Stimme auf der Diskursebene in den ausgewählten Werken analysieren.

\section{Arno Geiger: Der alte König in seinem Exil}

Arno Geiger erzählt in seinem Buch Der alte König in seinem Exil über seinen Vater August, der an Alzheimer erkrankt ist und an dessen Betreuung, Versorgung und Pflege sich alle vier Kinder, die getrennt lebende Ehefrau und im Haus lebende Pflegerinnen intensiv beteiligen. Geigers Text ist stark autobiografisch geprägt ${ }^{3}$ und wird von einem Ich-Erzähler erzählt. Mit der Frage des Erzählers beschäftigen sich

3 Vgl. Bestseller-Autor Arno Geiger: "Das Ende des Lebens ist auch Leben". https://www.spiegel.de/kultur/literatur/bestseller-autor-arno-geiger-das-ende-des-lebens-ist-auch-lebena-745909.html. Zugang am 5. Mai 2021. 
Martínez und Scheffel ${ }^{4}$ im Rahmen der Kategorie der Stimme und behandeln dabei, neben der Person des Erzählers, auch den Akt des Erzählens und das Verhältnis von Erzähler und Erzähltem (2016: 72).

Im Buch Der alte König in seinem Exil erfolgt die Erzählung auf der ersten Ebene und sie kann deswegen als extradiegetisch (Martínez \& Scheffel, 2016: 80) bezeichnet werden. Da die Erzählung von einem Ich-Erzähler erzählt wird, spricht man von einem homodiegetischen Erzähler, weil der Erzähler an der von ihm erzählten Geschichte als Figur beteiligt ist (Martínez \& Scheffel, 2016: 86). Der Ich-Erzähler der Erzählung heißt Arno Geiger, was darauf hinweist, dass dieser Ich-Erzähler mit dem Autor Arno Geiger identisch ist. Die Ich-Erzählform ist eine spezifische Erzählform, in der zwischen erzählendem und erlebendem Ich (Vogt, 1998: 71; Petersen, 1993: 56; Martínez \& Scheffel, 2016: 86) unterschieden wird. Obwohl es sich in solchen Texten immer um die eine und selbe Ich-Person handelt, betont J. Petersen den „Doppelaspekt der IchForm" (1993: 56) und hebt hervor, dass es zwischen erzählendem und erlebendem Ich eine Differenz gibt und dass „das Erzählen eines Ich-Erzählers einen Doppelaspekt erhält: es lässt den Erzählenden ebenso erkennbar werden wie den Erlebenden, das Erzählen wird bipolar" (Petersen, 1993: 56). Über diesen Doppelaspekt der Ich-Form schreibt J. Vogt folgendes:

Das Wechselspiel von erzählendem und erlebendem Ich [...] drückt eine Differenz und Spannung, wo nicht gar einen Bruch in der Identität des Erzählers aus: Er schreibt aus der Rückschau, ist gealtert und kennt mindestens teilweise die Folgen des vergangenen Geschehens. Aufgrund seiner Lebenserfahrung, veränderter Auffassungen und Maßstäbe betrachtet er das Tun und Treiben seines früheren Ich mit Abscheu, Kritik, Ironie oder auch Nachsicht, jedenfalls mit Distanz. (Vogt, 1998: 71)

Dieses Wechselspiel von erzählendem und erlebendem Ich lässt sich sehr gut in Geigers Erzählung analysieren. Schon am Anfang schildert der Erzähler Arno Geiger, d. h. das erzählende Ich, wie wenig die Figur Arno Geiger seiner Erzählung, also das erlebende Ich, und seine Familie von der Krankheit des Vaters wussten.

Die Schatten der Anfänge verfolgen mich noch immer, obwohl die Jahre einen gewissen Abstand hergestellt haben. Wenn ich aus dem Fenster hinunter auf den winterstarren Obstgarten schaue und daran zurückdenke, was mit uns passiert ist, überkommt mich das Gefühl eines vor langer Zeit begangenen Fehltritts.

\footnotetext{
${ }^{4}$ Jochen Vogt nennt dieses Kapitel Die typischen Erzäh/situationen, Jürgen Petersen Erzählformen.
} 
Die Krankheit des Vaters fing auf so verwirrenden Weise langsam an, dass es schwierig war, den Veränderungen die richtige Bedeutung beizumessen. [...]

Die frühesten Anzeichen der Krankheit zeigten sich Mitte der neunziger Jahre, doch gelang es uns nicht, die Ursache richtig zu deuten. Mit bitterem Kopfschütteln erinnere ich mich an die Renovierung der Terrassenwohnung, als der Vater die Betondeckel der ehemaligen Klärkammern zerschlug, weil er die Deckel alleine nicht hochheben und zurück in die Öffnung legen konnte.

[...] Niemand sah, dass er langsam seine alltagspraktischen Fähigkeiten verlor.

Die Krankheit zog ihr Netz über ihn, bedächtig, unauffällig. Der Vater war schon tief darin verstrickt, ohne dass wir es merkten. (Geiger, 2011: 19-20)

Aus den zitierten Sätzen kann man sehen, wie das erzählende Ich das Verhalten des erlebenden Ich dem kranken Vater gegenüber empfindet. Die genannte Stelle lässt sich sehr leicht mit den oben angeführten Worten Jochen Vogts in Verbindung bringen. Das erzählende Ich schreibt bzw. erzählt aus der Rückschau, ist gealtert und kennt die Folgen des vergangenen Geschehens. Im Moment, als das erzählende Ich diese Sätze schreibt, sind die Jahre vergangen, in denen der Vater und zusammen mit ihm auch sein Sohn Arno, das erlebende Ich, und die ganze Familie mit der Krankheit kämpfen. Das erlebende Ich der neunziger Jahre des 20. Jahrhunderts verstand nicht, dass etwas Ungewöhnliches mit dem Vater passiert und dass er krank ist, während das erzählende Ich aus dem ersten Jahrzehnt des neuen Jahrhunderts Zeit hatte, über diese Lebensperiode nachzudenken und da es aus der Rückschau schreibt, kann er dank der Lebenserfahrung das Verhalten seines früheren Ich, des erlebenden Ich, kritisch betrachten. Diese Rückschau ermöglicht ihm, sich der Situation anzupassen und sie zu akzeptieren.

Am Anfang waren diese Anpassungsmaßnahmen schmerzhaft und kräftezehrend. [...] Doch mittlerweile habe ich die neue Rolle einigermaßen gut hineingefunden. Und ich habe es auch gelernt, dass man für das Leben eines an Demenz erkrankten Menschen neue Maßstäbe braucht. [...] Für ihn gibt es keine Welt außerhalb der Demenz. Als Angehöriger kann ich deshalb nur versuchen, die Bitterkeit des Ganzen ein wenig zu lindern, indem ich die durcheinandergeratene Wirklichkeit des Kranken gelten lasse. (Geiger, 2011: 10-11)

An vielen Stellen in Geigers Text findet man reflexive und selbstreflexive Bemerkungen. Der Erzähler reflektiert sehr oft über sein Verhalten und über das Verhalten der ganzen Familie, wobei sie sehr lange nicht in der Lage waren, einzusehen, worum es geht. 
Da der Vater schon immer einen Hang zum Eigenbrötlerischen hatte, erklärten wir uns seine bald nach der Pensionierung auftretenden Aussetzer damit, dass er jetzt Anstalten machte, jegliches Interesse an seiner Umwelt zu verlieren. Sein Verhalten erschien typisch für ihn. Also gingen wir ihm etliche Jahre mit Beschwörungen auf die Nerven, er solle sich zusammenreißen.

Heute befällt mich ein stiller Zorn über diese Vergeudung von Kräften; denn wir schimpften mit der Person und meinten die Krankheit. [...] Er wollte dem Vergessen nicht trotzen, verwendete nie auch nur die geringsten Gedächtnisstützen und lief daher auch nicht Gefahr, sich zu beklagen, jemand mache Knoten in seine Taschentücher. Er leistete sich keinen hartnäckigen Stellungskrieg gegen seinen geistigen Verfall, und er suchte nicht ein einziges Mal das Gespräch darüber, obwohl er - aus heutiger Sicht - spätestens Mitte der neunziger Jahre um den Ernst der Sache gewusst haben muss. Wenn er zu einem seiner Kinder gesagt hätte, tut mir leid, mein Gehirn lässt mich im Stich, hätten alle besser mit der Situation umgehen können. So jedoch fand ein jahrelanges Katz- und Maus-Spiel statt, mit dem Vater als Maus, mit uns als Mäusen und mit der Krankheit als Katze. (Geiger, 2011: 7-8)

Aus der Sicht des erzählenden Ich besserte sich die Situation, als die Familie begriffen hat, was mit dem Vater geschieht. Gleichzeitig damit kam die Enttäuschung wegen der zu späten Reaktion und die Bitterkeit wegen der verpassten Zeit.

Schrecklich ist vor allem, was wir nicht begreifen. Deshalb besserte sich die Situation, als immer mehr Anzeichen darauf hindeuteten, dass den Vater mehr als nur Vergesslichkeit und Motivationsprobleme plagen. Dass ihn ganz alltägliche Dinge vor unlösbare Probleme stellten, ließ sich mit Zerstreutheit nicht mehr erklären, unmöglich, sich noch länger zu täuschen. In der Früh zog er sich nur halb, verkehrt oder vierfach an, mittags schob er die Tiefkühlpizza mitsamt der Verpackung ins Rohr, und seine Socken deponierte er im Kühlschrank. Auch wenn wir das ganze Ausmaß des Schreckens weiterhin nur langsam erfassten, war uns irgendwann doch klar, der Vater lässt sich nicht hängen, sondern leidet an Demenz. [...]

Die Einsicht in den wahren Sachverhalt bedeutete für alle eine Erleichterung. Jetzt gab es für das Chaos der zurückliegenden Jahre eine Erklärung, die wir akzeptieren konnten, wir fühlten uns nicht mehr so am Boden zerstört. Nur die Einsicht, dass wir viel zu viel Zeit damit vergeudet hatten, gegen ein Phantom anzukämpfen, war bitter - Zeit, die wir tausenmal sinnvoller hätten nutzen sollen. Wenn wir klüger, aufmerksamer und interessierter gewesen wären, hätten wir nicht nur dem Vater, sondern auch uns selber vieles erspart, und vor allem hätten wir besser auf inn aufpassen und noch rasch einige Fragen stellen können. (Geiger, 2011: 25-26)

Der alte König in seinem Exil ist in Bezug auf den Zeitpunkt des Erzählens eine Kombination aus späterem und gleichzeitigem Erzählen (Martínez \& Scheffel, 2016: 
73). Der Ich-Erzähler erzählt die Geschichte des Vaters im Präteritum, was genügt, um sie als das spätere Erzählen zu kategorisieren. Außerdem tritt in diesen Textpassagen das erlebende Ich der Vergangenheit in den Vordergrund und erzählt von den Ereignissen aus der Vergangenheit. Die Reflexionen des Ich-Erzählers aus der Gegenwart können dem Typ des gleichzeitigen Erzählens zugeschrieben werden. Am Anfang des Erzählens ist das erzählte Geschehen, d. h. Augusts Lebensgeschichte und seine Krankheit, noch nicht abgeschlossen, und der Zeitabstand zwischen dem Erzählten und dem Erzählen verringert sich mit der Zeit. Das erzählende Ich unterbricht sehr oft seine Erzählung und wird zum erlebenden Ich, zur handelnden Figur seiner Erzählung, die aus heutiger Sicht, aus der Sicht des erzählenden Ich, über die Geschehnisse in der Vergangenheit reflektiert. Mit der Zeit verschwimmt die Distanz und die Grenze zwischen erzählendem und erlebendem Ich, d. h. zwischen schreibendem Erzähler und handelndem Helden. Das erlebende Ich auf der ersten Seite von Geigers Erzählung ist sechs Jahre alt, am Ende der Erzählung ist das erlebende Ich identisch mit dem erzählenden Ich und stellt fest: "Zum Zeitpunkt, da ich diese Sätze schreibe, bin ich fast genau halb so alt wie er" (Geiger, 2011: 189).

Im Kapitel Stellung des Erzählers zum Geschehen ${ }^{5}$ der Kategorie der Stimme besprechen Martínez und Scheffel (2016: 86-87) auch den Grad der Beteiligung des Erzählers am erzählten Geschehen. Aus allen zitierten Stellen kann man sehen, dass der Ich-Erzähler in Geigers Text stark am erzählten Geschehen beteiligt ist, so dass wir schließen können, dass es sich in diesem Fall um eine der Hauptfiguren handelt. Die Hauptfigur ist der kranke Vater, aber die Erzählung wird nicht von ihm, sondern von seinem Sohn präsentiert. Das bedeutet, dass ein Mitglied der Familie von der Krankheit erzählt, und nicht der Erkrankte selbst, woraus man die Folgerung ziehen kann, dass die Krankheit durch einen Vermittler und nicht durch den Erkrankten wahrgenommen und dargestellt wird. So notiert das erzählende Ich über die Demenzerkrankung des Vaters:

Ich stelle mir Demenz in der mittleren Phase, in der sich mein Vater momentan befindet, ungefähr so vor: Als wäre man aus dem Schlaf gerissen, man weiß nicht, wo man ist, die Dinge kreisen um einen her, Länder, Jahre, Menschen. Man versucht sich zu orientieren, aber es gelingt nicht. Die Dinge kreisen weiter. Tote, Lebende, Erinnerungen, traumartige Halluzinationen, Satzfetzen, die einem nichts sagen - und dieser Zustand ändert sich nicht mehr für den Rest des Tages. (Geiger, 2011: 8-9)

\footnotetext{
${ }^{5}$ Jochen Vogt bearbeitet dieselbe Problematik im Kapitel Die typischen Erzäh/situationen, Jürgen Petersen im Kapitel Erzählformen.
} 
Die zweite Kategorie des, Wie' eines Erzähltextes ist die Kategorie der Zeit. Innerhalb dieser Kategorie lässt sich, nach Genette, "das Verhältnis zwischen der Zeit der erzählten Geschichte und der Zeit der Erzählung im Sinne der drei Fragen In welcher Reihenfolge?, Wie lange? und Wie oft? systematisieren" (Martínez \& Scheffel, 2016: 34). Was die Frage der Ordnung (In welcher Reihenfolge?) angeht, bezieht sie sich auf "die Abfolge eines Geschehens in der Zeit und die Abfolge seiner Darstellung im Rahmen der Erzählung" (Martínez \& Scheffel, 2016: 35). Wenn die chronologische Ordnung einer Ereignisfolge nicht erhalten ist, spricht man von einer narrativen Anachonie. Die kann in unterschiedlichen Formen auftreten (Martínez \& Scheffel, 2016: 35).

Geigers Text beginnt mit einer Analepse ${ }^{6}$, der Erzähler erinnert sich an seinen längst verstorbenen Großvater. Eine Besonderheit dieser Analepse ist die, dass sie gleichzeitig als eine Prolepse ${ }^{7}$ fungiert.

Als ich sechs Jahre alt war, hörte mein Großvater auf, mich zu erkennen. Er wohnte im Nachbarhaus unterhalb unseres Hauses, und weil ich seinen Obstgarten als Abkürzung auf dem Weg zur Schule benutzte, warf er mir gelegentlich ein Scheit Holz hinterher, ich hätte in seinen Feldern nichts verloren. Manchmal jedoch freute inn mein Anblick, er kam auf mich zu und nannte mich Helmut. Das war ebenfalls nichts, womit ich etwas anfangen konnte. Der Großvater starb. Ich vergaß diese Erlebnisse - bis die Krankheit bei meinem Vater losging. (Geiger, 2011: 7)

Berücksichtigt man die Stellung, die Funktion und den Status dieser Anachronie lässt sich feststellen, dass der Text mit dieser Passage anfängt und dass sie als eine Art Exposition verstanden werden kann. In diesem Sinne handelt es sich hier um eine aufbauende Rückwendung (Lämmert, 1955: 104-108), in der die Symptome der Demenz bei dem Großvater beschrieben wurden. Diese Rückwendung beinhaltet zugleich, im letzten Satz, eine Vorausdeutung auf das zukünftige Schicksal des Vaters, d. h. "auf ein Geschehen, das im Augenblick des Erzählens noch zur Zukunft der erzählten Geschichte gehört" (Martínez \& Scheffel, 2016: 39). In diesem Fall geht es um eine einführende Vorausdeutung (Martínez \& Scheffel, 2016: 39), die zugleich als eine zukunftsgewisse Vorausdeutung (Lämmert, 1955: 143-175) funktioniert. Der Erzähler vermittelt dem Leser aus einem größeren zeitlichen Abstand die Information

6 E. Lämmert nennt diesen Typ der Anachronie Rückwendung. Er schreibt ausführlich über die Rückwendungen und Vorausdeutungen (1955: 100-192).

7 In Lämmerts Therminologie Vorausdeutung. 
über die Krankheit des Vaters in der Zukunft und macht den Leser auf diese Weise zum Mitwisser der Zukunft.

Arno Geiger strukturiert den Text so, dass er zeitraffendes bzw. summarisches (Raffung) und zeitdeckendes (Szene) Erzählen (Martínez \& Scheffel, 2016: 42) kombiniert. Um die Lebensgeschichte des Vaters nicht nur auf die Zeit der Krankheit zu reduzieren und um die Persönlichkeit des Vaters zu schildern, stellt der Erzähler die Lebensphasen von August Geiger (Kindheit, Jugend, Heirat, Geburt der Kinder, Scheidung von der Ehefrau) mehr oder weniger gerafft, bzw. summarisch dar. In diese summarische Erzählung integriert er immer wieder kurze Episoden, meist Gespräche mit dem Vater, in Form von zeitdeckenden Darstellungen. Diese szenischen Darstellungen sind sehr oft in der Form der zitierten Figurenrede (Martínez \& Scheffel, 2016: 54) angeführt. Die Wahl eines solchen Verfahrens ermöglicht dem Erzähler, die Rede des alten Mannes genau wiederzugeben bzw. zu zitieren. Durch die Verwendung der direkten Rede erhöht sich der Grad an Authentizität und Unmittelbarkeit und vermindert sich die Distanz zwischen Leser und Geschehen. Dass Geiger eben dem Aspekt der Sprache große Aufmerksamkeit schenkt, hat mit der Art der Krankheit zu tun, besonders, wenn man weiß, dass die Alzheimer-Krankheit wesentliche Spuren in der Sprache eines an Alzheimer erkrankten Menschen hinterlässt. Das bedeutet, dass sich mit Fortschreiten der Krankheit auch die Sprache des Erkrankten verändert.

Laut Definition ist die Alzheimer-Krankheit "die häufigste Form der Demenz und eine unheilbare Störung des Gehirns" (https://www.alzheimerforschung.de/alzheimer/, Zugang am 15. Mai 2021). Die Betroffenen werden zunehmend vergesslich, verwirt und orientierungslos und bei ihnen ändert sich im Verlauf der Erkrankung auch die Persönlichkeit und das Verhalten. Viele Patienten werden unruhig, aggressiv und depressiv. Das Urteilsvermögen und die Sprachfähigkeit lassen nach (ebenda). Als typische Symptome der AlzheimerKrankheit gelten Störungen: "des Gedächtnisses, der Sprache, des planerischen Denkens und Handelns, der Wahrnehmung, der Alltagskompetenz und der örtlichen und zeitlichen Orientierung" (https://www.alzheimerforschung.de/alzheimer/symptome/, Zugang am 15. Mai 2021). Was die Sprachprobleme angeht, "fällt es vielen Erkrankten schwer, einer Unterhaltung zu folgen und aktiv an einem Gespräch teilzunehmen. Sie verlieren den Faden, benutzen unpassende Füllwörter oder haben Wortbildungsprobleme" (https://www.alzheimerforschung.de/alzheimer/symptome/frueherkennung/, Zugang am 15. Mai 2021). 
An einigen Stellen in seiner Erzählung schildert Geiger, wie sich der Vater in Bezug auf die Sprache verändert. Seine Sprachdefizite sind im Umgang mit anderen Menschen nicht auffallend, wenn es um kurze und einfache Gespräche geht. In allen anderen Fällen werden seine Sprachprobleme offensichtlich.

Wenn man ihn auf der Straße traf, kam man zunächst nicht auf die Idee, dass etwas mit inm nicht stimmte. Er strahlte unterschiedslos jeden Menschen an und lavierte sich mit kleinen Scherzen durch kurze Wortwechsel, so dass die Leute behaupteten, er erkenne sie immer, er sei derselbe Schelm wie eh und je. Erst wenn das Gespräch auf einen Gegenstand kam, der etwas mehr Zusammenhang und Übersicht verlangte, offenbarten sich seine Schwächen. (Geiger, 2011: 66-67)

Für die Alzheimer-Krankheit typische Symptome sind auch bei August Geiger erkennbar.

Aus zukünftig machte er kuhzünftig, das Ende des Lateins, das ich bekundete, konterte er, er selber befinde sich nicht am Ende des Lateins, sondern am Ende des Daseins. Dabei betonte er die Wörter so, dass die lautliche Verwandtschaft unüberhörbar war. Er verwendete Wörter wie pressant und pressiert, dawei und bistra. Auch einige Redensarten, die ich lange nicht gehört hatte, kamen wieder zum Vorschein:

„Das Leintuch ist nun einmal nicht größer, da hilft kein Ziehen." [...] Wenn ihm ein Wort nicht einfiel, sagte er:

"Ich weiß nicht, wie ich es taufen soll."

Locker fielen ihm die Wörter aus dem Mund, klack, klack. Er war entspannt, er redete, was inm einfiel, und was inm einfiel, war oft nicht nur originell, sondern hatte eine Tiefe, bei der ich mit dachte: Warum fällt mir so etwas nicht ein! Ich wunderte mich, wie präzise er sich ausdrückte und wie genau er den richtigen Ton traf und wie geschickt er die Wörter wählte. (Geiger, 2011: 101-102)

Diese Passage zeigt einerseits, dass August Geiger nicht imstande ist, einige Wörter grammatisch korrekt anzuwenden, aber auch andererseits Überraschung und Erstaunen des Sohnes, wie präzise und geschickt sich der Vater unter diesen Umständen ausdrücken kann. Die Situation verschlimmert sich aber mit der Zeit, so dass Geiger aus heutiger Sicht feststellt: „Schade nur, dass die Sprache langsam aus ihm heraussickert, dass auch die Sätze, bei denen einem vor Staunen die Luft wegbleibt, immer wieder seltener werden. Was da alles verlorengeht, das berührt mich" (2011: 11-12).

Geiger beschreibt auch andere Symptome der Alzheimer-Krankheit bei seinem Vater. Besondere Aufmerksamkeit widmet er der Orientierungslosigkeit und stellt viele Situationen dar, in denen der Vater sein eigenes Haus nicht mehr erkannte und ständig 
den Wunsch äußerte, nach Hause gehen zu wollen. Zu Beginn versuchten die Familienangehörigen ihm logisch zu erklären, dass er zu Hause ist. Erst später verstanden sie, dass er nicht mehr in der Lage war, logische Zusammenhänge zu begreifen. Ein Beispiel für eine solche Situation ist folgendes Gespräch zwischen dem Vater und seiner Tochter Helga.

Es muss um das Jahr 2004 gewesen sein, da erkannte er plötzlich sein eigenes Haus nicht mehr. Das geschah überraschend schnell, schockierend schnell, so dass wir es gar nicht fassen konnten. Lange Zeit weigerten wir uns zu akzeptieren, dass der Vater so etwas Selbstverständliches wie das eigene Haus vergessen hatte.

Eines Tages wollte sich meine Schwester sein Bitten und Drängen nicht länger anhören. Alle fünf Minuten sagte er, dass er zu Hause erwartet werde, das war nicht zum Aushalten. Unserem damaligen Empfinden nach überstiegen seine endlosen Wiederholungen jedes erträgliche Maß.

Helga ging mit inm hinaus auf die Straße und verkündete:

"Das ist dein Haus!"

"Nein, das ist nicht mein Haus", erwiderte er.

"Dann sag mir, wo du wohnst."

Er nannte die korrekte Straße mit Hausnummer.

Triumphierend zeigte Helga auf das Hausnummerschild neben der Eingangstür und fragte:

"Und, was steht hier?"

Er las ihr die zuvor genannte Adresse vor.

Helga fragte:

"Was schließen wir daraus?"

"Dass jemand das Schild gestohlen und hier angeschraubt hat", erwiderte der Vater trocken - was eine etwas phantastisch anmutende Deutung war, die aber keineswegs jede Schlüssigkeit vermissen ließ.

"Warum sollte jemand unser Hausnummerschild klauen und an sein Haus schrauben?", fragte Helga empört.

"Das weiß ich auch nicht. Die Leute sind halt so."

Das stellte er mit Bedauern fest, gleichzeitig zeigte er nicht den geringsten Anflug von Skepsis angesichts der Unwahrscheinlichkeit seiner Argumentation. (Geiger, 2011: 52-54)

Diese Textstelle ist zugleich ein sehr gutes Beispiel für die dritte Kategorie im Martínez' und Scheffels analytischen Beschreibungsmodell, die des Modus. In dieser Kategorie behandeln sie den Grad an Mittelbarkeit und die Perspektivierung des Erzählten bzw. Distanz und Fokalisierung (2016: 50). Der zitierte Ausschnitt kombiniert den narrativen und den dramatischen Modus (Martínez \& Scheffel, 2016: 52). Er beginnt in der Form des summarischen Erzählens von der Vergesslichkeit des Vaters und seinem Bitten und Drängen, nach Hause zu gehen. Das veranlasst seine Tochter, genervt durch seine endlosen Wiederholungen, ihn nach seiner Adresse zu 
fragen. Diese Situation ermöglicht den Übergang in die szenische Darstellung des Gesprächs zwischen dem Vater und der Tochter. Mit der Darstellung des Figurendialogs hält sich der Erzähler zurück, die Mittelbarkeit des Erzählens ist weniger sichtbar und auf diese Weise entsteht bei dem Leser die Illusion einer unmittelbaren Nähe zum erzählten Geschehen.

Geiger verwendet in seiner Erzählung sehr oft unterschiedliche Formen des dramatischen Modus. So beginnt jedes Kapitel mit einem kurzen Dialog zwischen dem Vater und dem Sohn. Diese Gespräche sind wörtlich wiedergegeben, ohne Kommentare des Erzählers, ohne verba dicendi und ohne distanzierende Anführungszeichen. In solchen Fällen reden Martínez und Scheffel von einem Extremfall einer Erzählung im dramatischen Modus und bezeichnen solche Fälle als autonome direkte Figurenrede (2016: 54). Die Rede der erlebenden Figuren ist gegenwärtig, die Distanz zum erzählten Geschehen ist vollkommen reduziert und die Anwesenheit der narrativen Instanz ist nicht sichtbar.

Aber, Papa! Hallo! Hallo! Das geht nicht! Erklär mir lieber, wie du nach Hause gehen willst, wenn du schon zu Hause bist.

Ich verstehe nicht ganz.

Du bist zu Hause und willst nach Hause gehen. Man kann doch nicht

nach Hause gehen, wenn man schon zu Hause ist.

Das ist sachlich richtig.

Und?

Das interessiert mich alles bei weitem nicht so sehr wie dich. (Geiger, 2011: 47)

In vielen Gesprächspassagen benutzt Geiger auch andere Möglichkeiten der Präsentation von gesprochener Rede. Im oben zitierten Gespräch mit Helga findet man neben der autonomen direkten Figurenrede auch die Beispiele der direkten Figurenrede (Martínez \& Scheffel, 2016: 54) und der erzäh/ten Figurenrede (Martínez \& Scheffel, 2016: 55) in Form des Erzählerberichts. Diese Szene ist ein interessantes Beispiel dafür, wie die verschiedenen Formen der Erzählung von Worten kombiniert werden und welche mimetische Effekte sie hervorrufen können.

\section{Eugen Ruge: In Zeiten des abnehmenden Lichts}

Eugen Ruge erzählt in seinem Erstlingroman über vier Generationen der Familie Umnitzer, aber auch zugleich über die Geschichte eines Staates, den es heute nicht mehr gibt, der Deutschen Demokratischen Republik. Sein Bestseller ist, ähnlich wie bei Geiger, stark autobiografisch geprägt, so dass man sagen kann, hier erzählt Ruge 
die Geschichte (s)einer Familie, (s)eines Landes und (s)eines Lebens. Anders als Geiger verzichtet er nicht auf die Gattungsbezeichnung, das Buch trägt den Untertitel Roman einer Familie. Schon auf den ersten Blick kann man feststellen, dass der Roman eine spezifische Struktur hat. Er ist in zwanzig Kapitel unterteilt und jedes Kapitel ereignet sich zu einem gewissen Zeitpunkt: es geht entweder um ein Jahr oder um ein bestimmtes Datum - den 1. Oktober 1989.

Der Autor kombiniert im Roman drei Erzähllinien, die sich auf unterschiedliche Zeitebenen beziehen und verbindet sie miteinander. Die erste erzäh/te Zeit (Martínez \& Scheffel, 2016: 33) ist die chronologische Erzählung der Familiengeschichte mit den Stationen 1952, 1959, 1961, 1966, 1973, 1976, 1979, 1991 und 1995. Herausgehoben aus dieser Chronologie ist der 1. Oktober 1989, Wilhelms 90. Geburtstag, an dem einige für die Familie wichtige Ereignisse passieren: Alexanders Flucht in die BRD, Wilhelms Vergiftung seitens Charlotte, Irinas erster Alkoholexzess. Dieser Tag wird sechsmal aus unterschiedlichen Figurenperspektiven ${ }^{8}$ präsentiert und die Geschehnisse dieses Tages werden im 3. Kapitel (aus Irinas Perspektive), im 7. (aus Nadjeshda Iwanownas Perspektive), im 9. (Wilhelms Perspektive), im 13. (Markus' Perspektive), im 16. (Kurts Perspektive) und im 19. Kapitel (Charlottes Perspektive) dargestellt. Dieses Verfahren ist ein typisches Beispiel des multiperspektivischen Erzählens (Martínez \& Scheffel, 2016: 70) und einer repetitiven Erzählung (Martínez \& Scheffel, 2016: 49). Die dritte Zeitebene bildet Alexanders Mexikoreise im Jahr 2001, die chronologisch in fünf Kapiteln (im 1., 5., 11., 15. und 20. Kapitel) erzählt wird und einen kritischen, zeitlich distanzierten Rückblick auf die erzählte Geschichte ermöglicht (vgl. https://de.wikipedia.org/wiki/In Zeiten des abnehmenden Lichts, Zugang am 28. Mai 2021). Im Rahmen dieses Beitrags konzentrieren wir uns nur auf das Thema Krankheit im genannten Werk.

Zwei Tage lang hatte er wie tot auf seinem Büffelledersofa gelegen. Dann stand er auf, duschte ausgiebig, um auch den letzten Partikel Krankenhausluft von sich abzuwaschen, und fuhr nach Neuendorf.

Er fuhr die A115, wie immer. Schaute hinaus in die Welt. Prüfte, ob sie sich verändert hatte. Und - hatte sie?

Die Autos kamen ihm sauberer vor. Sauberer? Irgenwie bunter. Idiotischer.

Der Himmel war blau, was sonst. (Ruge, 2011: 7)

${ }^{8}$ Ausführlicher dazu in Aćimović, 2016: 268-286. 
Mit diesen Sätzen beginnt Ruges Roman In Zeiten des abnehmenden Lichts. Schon zu Beginn des Romans erfährt der Leser, dass Alexander vor zwei Tagen aus dem Krankenhaus entlassen worden ist, was auf eine Krankheit impliziert. Die Verwendung des epischen Präteritums weist darauf hin, dass es sich hier um das spätere Erzählen handelt. Der Roman ist so strukturiert, dass die ersten zwei Erzählebenen aus der Sicht unterschiedlicher Figuren vermittelt werden. Die dritte Erzählebene wird aus Alexanders Sicht präsentiert, so dass die Erzählung durch eine interne Fokalisierung (Martínez \& Scheffel, 2016: 68) gekennzeichnet ist. Interne Fokalisierung bedeutet ",Mitsicht' - der Erzähler sagt nicht mehr, als die Figur weiß" (ebenda). Obwohl diese Textpassage in der dritten Person steht, ist es Alexanders Sichtweise die, die dem Leser dargeboten wird. Die Geschehnisse werden durch seine Wahrnehmung für den Leser erfahrbar. Franz K. Stanzel nennt eine solche Person „eine Reflektorfigur" (2001: 194) und definiert, dass eine Reflektorfigur "reflektiert, d. h. spiegelt Vorgänge der Außenwelt in ihrem Bewußtsein wider, nimmt wahr, empfindet, registriert, aber immer stillschweigend, denn sie 'erzählt' nie, das heißt, sie verbalisiert ihre Wahrnehmungen, Gedanken und Gefühle nicht, da sie sich in keiner Kommunikationssituation befindet" (Stanzel, 2001: 194). Nach ihm bestimmen die Dominanz einer Reflektorfigur, das Überwiegen der Innenperspektive und die am ErBezug auf die Reflektorfigur festzumachende Nicht-Identität der Seinsbereiche von Erzähler und Figuren die personale Erzäh/situation (Martínez \& Scheffel, 2016: 98). J. Petersen spricht auch von einem personalen Erzählverhalten und definiert es als „Perspektivismus der Darstellung, der die Sehweise der Figur in den Mittelpunkt rückt" (1993: 70).

Es scheint, als ob es in Ruges Text keinen Erzähler gäbe. Im Vordergrund steht nicht die Perspektive eines Erzählers, sondern die Wahrnehmungsperspektive einer am erzählten Geschehen unmittelbar beteiligten Figur, in diesem konkreten Fall Alexanders Perspektive. Hier gibt es keinen Narrator im Sinne einer Person, so dass man hier von einer "vermittelnden narrativen Instanz" (Martínez \& Scheffel, 2016: 53) sprechen kann. Im Hinblick auf die Parameter Ort des Erzählens und Stellung des Erzählers zum Geschehen handelt es sich hier um eine extradiegetischeheterodiegetische narrative Instanz, da sie nicht zu den Figuren ihrer Geschichte gehört.

Nachdem Alexander zu seinem Vater Kurt gekommen ist, was in der Form des zeitraffenden Erzählens im narrativen Modus dargestellt wird, wechselt die Erzählung in die Form des zeitdeckenden Erzählens im dramatischen Modus. Da Alexanders 
Wahrnehmungsperspektive im Vordergrund steht, hat der Leser auch den Einblick in seine Gedanken in der Form eines „Bewusstseinsberichts” (Martínez \& Scheffel, 2016: 59): „Obwohl er einen Schlüssel hatte, klingelte er. Testen, ob Kurt aufmachte sinnlos. Ohnehin wusste er, dass Kurt nicht aufmachen würde" (Ruge, 2011: 8). Der schnelle Wechsel von zeitraffendem und zeitdeckendem Erzählen kennzeichnet den narrativen Rhythmus und deutet auf Kurts Erkrankung an. Direkte Figurenrede im dramatischen Modus verringert die Distanz zum erzählten Geschehen und reduziert vollkommen die Präsenz der narrativen Instanz.

- Mach auf, rief Alexander.

Kurt kam näher, glotzte.

- Mach auf!

Aber Kurt rührte sich nicht.

Alexander schloss auf, umarmte seinen Vater, obwohl ihm die Umarmung seit langem unangenehm war. Kurt roch. [...]

- Erkennst du mich, fragte Alexander.

- Ja, sagte Kurt. (Ruge, 2011: 8)

Danach werden nochmals Alexanders Gedanken wiedergegeben, diesmal in der Form eines längeren Gedankengangs, des Selbstgesprächs durch eine inquit-Formel in Gestalt eines verbum credendi eingeleitet (Martínez \& Scheffel, 2016: 64).

Eigentlich ein Witz, dachte Alexander, dass Kurts Verfall ausgerechnet mit der Sprache begonnen hatte. Kurt, der Redner. Der große Erzähler. [...] Egal, was Kurt erzählte [...] immer hatte es eine Pointe, immer hatte es Witz. Hatte gehabt. Fernste Vergangenheit. Der letzte Satz, den Kurt zusammenhängend hatte sagen können, war: Ich habe die Sprache verloren. Auch nicht schlecht. Verglichen mit seinem heutigen Repertoire eine Glanznummer. Doch das war vor zwei Jahre her: Ich habe die Sprache verloren. Und die Leute hatten wirklich gedacht, sieh mal an, er hat die Sprache verloren, aber sonst ... Sonst schien er noch einigermaßen beisammen zu sein. Lächelte, nickte. Zog Grimassen, die irgendwie passten. Verstellte sich schlau. Nur hin und wieder unterlief ihm Sonderbares: dass er Rotwein in seine Kaffetasse goss. Oder auf einmal ratlos mit einem Korken dastand - und ihn schließlich ins Bücherregal steckte. (Ruge, 2011: 10)

Durch Alexanders Gedanken erfährt der Leser von Kurts Demenzerkrankung. An dieser Stelle können gewisse Parallele zu Geigers Text gezogen werden: beide Väter zeigen Sprachdefizite - bei Kurt, der vor zwei Jahren "die Sprache verloren hat" und „Ja das einzige Wort war, das er noch beherrschte" (Ruge, 2011: 16) geht Verfall schneller und auffallender als bei August Geiger, der schon "über einem Jahrzehnt" (Geiger, 2011: 129) mit Alzheimer kämpft; beide haben Probleme mit der Bewältigung 
alltäglicher Tätigkeiten; beide sind auf die Hilfe anderer angewiesen. In beiden Fällen wird die Krankheit nicht aus der Perspektive des Erkrankten sondern aus der Sicht des Sohnes, d. h. mittelbar präsentiert.

Durch den schnellen Wechsel von zeitraffendem und zeitdeckendem Erzählen und die weitere Wiedergabe von Alexanders Gedanken entdeckt man, dass er vier Wochen lang im Krankenhaus war, wo ihm eine Krebserkrankung diagnostiziert wurde, "Non-Hodgkin-Lymphom, langsam wachsender Typ. Gegen das es - wie taktvoll ausgedrückt! - bis heute keine wirksame Therapie gebe" (Ruge, 2011: 25). Das dominant zeitraffende Erzählen bis zum Ende des Kapitels kennzeichnet etwas langsameres Erzähltempo, das Gedanken an die Krankheit unterbrechen: „Vergänglich - klang gut. Solange man keinen Krebs hatte" (Ruge, 2011: 22), "Und was, wenn sie sich geirrt hatten?" (Ruge, 2011: 23), "Nein, sie hatten sich nicht geirrt. Es gab ein Röntgenbild. Es gab ein CT. Es gab Laborwerte" (Ruge, 2011: 25). Der ständige Wechsel weist auf Alexanders innere Unruhe hin. Diese Unruhe ist mit der Nachricht über die Krankheit verbunden und die Gedanken an die Krankheit werden unmittelbar aus der Sicht des Erkrankten vermittelt. Das gleiche Erzählverfahren wurde auch im weiteren Text verwendet.

Das Betrachten des Telefons im Zimmer seiner Mutter ruft Erinnerungen an ihr letztes Gespräch hervor, die als Analepse in der direkten Rede angeführt wird:

- Saschenka. Du. Musst. Kommen.

Und nach jedem Wort ein langes, atmosphärisches Knistern, so dass man versucht war aufzulegen, weil man glaubte, die Leitung sei unterbrochen.

Und er? Was hatte er gesagt?

- Ich komme, wenn du aufgehört hast zu trinken. [...]

Oder hatte er "saufen" gesagt? Ich komme, wenn du aufgehört hast zu saufen? (Ruge, 2011: 24)

Mit dieser Analepse und mit der Art, wie sie präsentiert wird, wird auf die Abhängigkeit seiner Mutter Irina von Alkoholismus angedeutet. Irinas Alkoholismus begann am Tag von Alexanders Flucht in die BRD, am 1. Oktober 1989. Aus der Perspektive von Irinas Mutter, Nadjeshda Iwanowna, erfährt der Leser auch über Irinas Probleme mit Alkohol.

War trotzdem kein Grund zum Trinken, fand Nadjeshda Iwanowna. Gehörte sich einfach nicht für eine Frau, wo gab es denn so was, die Frau trinkt, und der Mann ist nüchtern, man schämte sich wirklich, rauchen rauchte sie auch, das war alles nicht richtig, sich betrinken zu Wilhelms 
Geburtstag, als ob Sascha zurückkäme, wenn sie sich da oben betrank. (Ruge, 2011: 148)

Kurt reflektiert ausführlicher. In seinen Gedanken tauchen Irinas Worte direkt transponiert, was ein Beipiel für die erlebte Rede (Martínez \& Scheffel, 2016: 55) ist.

Es war zwecklos, mit Irina zu diskutieren. [...] Hinzu kam der Alkohol - ein Stoff, dem die ruhsische Selle ohnehin in einem besonderen Maße zugeneigt schien. Zwar hatte Irina, anders als er selbst, schon von jeher kräftig getrunken, allerdings war es bisher immer eine Art "gesellschaftliches" Trinken gewesen. Dass sie sich in ihr Zimmer zurückzog und sich, Wyssozki hörend, in aller Einsamkeit betrank, war eine ziemlich neuartige Erscheinung. Gewiss war sie keine Alkoholikerin: Manchmal trank sie tagelang oder sogar wochenlang nicht. Und doch beunruhigte es Kurt, wenn er an die schier unbeherrschbare Kettenreaktion dachte, die ein einziger Kognak bei ihr auslösen konnte.

Diesen einen einzigen Kognak hatte Kurt ihr - nach der Nachricht von Saschas Flucht - nicht verwehren können. Aber kaum dass sie diesen einen einzigen Kognak getrunken hatte, hatte sie mit Vehemenz einen zweiten (und letzten) verlangt. [...] Den dritten Kognak goss sie sich selber ein und drohte fast handgreiflich zu werden, als Kurt ihr die Flasche wegnehmen wollte. (Ruge, 2011: 323-324)

Im 17. Kapitel wird unmittelbar aus Irinas Perspektive über ihre Alkoholsucht reflektiert. Während sie eine Klostergans zu Weihnachten vorbereitet und den Nachrichten im Radio folgt, kommt ihre Süchtigkeit zum Vorschein. Das zeitraffende Erzählen, im dem ihre übertriebene Mutterliebe und ihr Kummer wegen Alexanders Abwesenheit in den Vordergrund treten, wird ständig durch Gedankenzitate unterbrochen: „Ein Kognak täte gut, dachte Irina" (Ruge, 2011: 352); "O ja, ein Kognak täte jetzt gut, dachte sie, um das Gehirn ein bisschen von den immer gleichen Gedanken abzubringen ... aber sie hatte sich vorgenommen, heute nicht zu trinken" (Ruge, 2011: 353); [...] dann, dachte Irina, würde sie sich einen Kognak genehmigen - nur einen! - und jene Stunde genießen, die zu Weihnachten immer die schönste gewesen war:" (Ruge, 2011: 355). Durch dieses Erzählverfahren wird die Distanz zum erzählten Geschehen aufgehoben und der Leser hat das Gefühlt, sehr nahe am Geschehen zu sein. Das langsame Erzähltempo vermittelt den Eindruck des zeitdeckenden Erzählens. Mehrmalige Wiederholungen heben ihre Abhängigkeit hervor: "Sie kostete das Obst-Whisky-Gemisch - schmeckte nicht schlecht, aber eigentümlich" (Ruge, 2011: 356); "Nun musste sie wenigstens einmal kosten, wie das Zeug pur schmeckte" (Ruge, 2011: 357); "Und dann nahm sie das Fruchtsaft-WhiskyGemisch - und trank es. Erstens war es kein richtiger Alkohol. Zweitens ärgerte sie 
sich" (Ruge, 2011: 357). "Sie goss sich noch einen winzigen Schluck ein. [...] Den einen! Dann, versprochen, stellte sie die Flasche in die Speisekammer. Aber diesen einen brauchte sie jetzt:" (Ruge, 2011: 359); "Sie trank den Whisky - das Zeug drehte ganz schön! - und rauchte noch eine Zigarette an" (Ruge, 2011: 364), bis sie am Ende des Kapitels volltrunken auf den Küchenboden fällt.

Während Alexanders Krebserkrankung und Irinas Alkoholsucht aus der Wahrnehmungsperspektive der erlebenden Figuren präsentiert werden, wird Kurts Demenz nur aus Alexanders Sicht dargestellt. Wilhelms Demenz demgegenüber wird aus unterschiedlichen Perspektiven gezeigt. Sie wird zuerst durch Charlottes Worte an Irinas Mutter angedeutet: „- - Verstehen Sie, Nadjeshda Iwanowna, er ist schon ganz und gar ... Und dann machte sie eine schwer zu deutende Handbewegung. Was war mit Wilhelm? Ging es inm nicht gut" (Ruge, 2011: 152)? Im ersten Augenblick verstand sie nicht, was Charlotte damit gemeint hat, aber nach dem kurzen Gespäch mit inm, stellt sie fest, dass er krank ist: „Nadjeshda Iwanowna nickte - so stand es also um ihn. Hatte sich auf den Weg gemacht, der alte Wilhelm. Jetzt verstand sie das Dunkle in seinem Blick, sie hatte es schon gesehen, bei Todgeweihten" (Ruge, 2011: 153). Ihre Sicht auf seine Krankheit wird durch Wilhelms Wahrnehmung ergänzt.

Manchmal vergaß er, was zu tun war. Es kam inm so vor, als sei er über Nacht erstarrt. [...] Er ging in sein Zimmer und schloss die Tür. Wusste auf einmal nicht weiter - wieder so ein Moment. Wahrscheinlich lag es an den Tabletten. Schon seit einer Weile hegte er diesen Verdacht. Die Starre in den Gelenken. Die Leere im Kopf. Wer weiß, was für ein Zeug sie inm gab. Die Tabletten machten ihn dumm. Die Tabletten machten ihn vergesslich. Sie machten ihn so vergesslich, dass er am Morgen vergaß, dass er sich am Abend vorgenommen hatte, keine Tabletten zu nehmen.

Die Angst, das Gedächtnis zu verlieren. Wilhelm versuchte sich zu erinnern, probehalber: aber an was? (Ruge, 2011: 187-189)

Durch Wilhelms Gedanken, die Vergesslichkeit und Orientierungslosigkeit verraten, wird auf die Symptome der Demenz angedeutet. Im 9. Kapitel, in welchem aus seiner Perspektive auf die Ereignisse seines 90. Geburtstags reflektiert wird, sind auch seine Sprachdefizite auffällig, da er nicht mehr imstande ist, seine Gedanken in vollständigen Sätzen auszudrücken, was im Text durch die direkte Rede und sehr kurze und unvollständige Sätze zum Vorschein kommt.

Charlottes Perspektive vermittelt noch einen Blick auf Wilhelms Krankheit. Es wird geschildert, wie sie von dem Arzt mehrmals verlangte, Wilhelm in ein Pflegeheim zu schicken, was dieser ablehnte, weil ihm „handfeste Fakten" (Ruge, 2011: 390) 
fehlten. Durch einen langen Bewusstseinsbericht werden Wilhelms Krankheitsprobleme präsentiert, die er seinerseits nicht ernst nahm und verneinte.

Obwohl das multiperspektivische Erzählen die Möglichkeit eröffnet, die aus unterschiedlichen Perspektiven dargestellten Ereignisse unterschiedlich zu bewerten, ist hier ganz klar, dass alle Reflektorfiguren Wilhelms Demenz auf eine und dieselbe Weise reflektieren.

\section{Schlussfolgerung}

In diesem Beitrag wird anhand narratologischer Kriterien untersucht, auf welche Weise zwei zeitgenössische deutschsprachige Autoren das Thema Krankheit darstellen. Es lassen sich gewisse Parallelen zwischen den analysierten Texten ziehen. Beide Texte wurden im gleichen Jahr veröffentlicht und beide Texte weisen autobiografische Merkmale auf. Die Analyse hat gezeigt, dass die Autoren unterschiedliche erzählerische Mittel verwenden, um eine gezielte Wirkung hervorzurufen.

Geigers Text kombiniert das spätere und gleichzeitige Erzählen, ist durch viele Reflexionen des Ich-Erzählers gekennzeichnet, welcher die Geschichte des kranken August Geiger aus der Sicht seines Sohnes erzählt. Das Erzählen wechselt oft aus dem zeitraffenden Erzählen im narrativen Modus ins zeitdeckende Erzählen im dramatischen Modus, wodurch die Distanz zum erzählten Geschehen vermindert und die mimetische Illusion geweckt wird, nahe am erzählten Geschehen zu sein. Sehr oft wird die direkte Rede verwendet. Das Thema der Alzheimererkrankung des Vaters steht im Vordergrund der erzählten Geschichte. Die Krankheit wird nicht von dem Erkrankten sondern von einem Familienangehörigen, in diesem konkreten Fall, von seinem Sohn präsentiert.

Ruge wählt für seinen Roman eine spezifische Struktur in der Form von drei Erzählebenen, die ineinander verflochten sind. Spezifisch ist darüber hinaus auch die Präsentation der geschilderten Ereignisse, die aus der Sicht der unterschiedlichen Reflektorfiguren reflektiert werden. Dabei tritt die vermittelnde narrative Instanz in den Hintergrund. Ruge erzählt die Geschichte einer Familie und eines Staates, so dass bei ihm das Thema der Krankheit nicht im Vordergrund steht. An Demenz leiden Kurt Umnitzer und sein Stiefvater Wilhelm Powileit, wobei Ruge ähnlich wie Geiger, einige von den Symptomen der Demenzerkrankung zur Sprache bringt, wie z. B. Sprachprobleme, Gedächtnisverlust, Orientierungslosigkeit. Über Kurts Demenz erfährt der Leser ähnlich wie bei Geiger nur aus der Sicht seines Sohnes, während über 
Wilhelms Demenzerkrankung aus unterschiedlichen Perspektiven reflektiert wird. Angesprochen werden auch Alexanders Krebserkrankung und Irinas Alkoholsucht. Ruge verwendet ähnliche erzählerische Mittel wie Geiger: das spätere Erzählen, der schnelle Wechsel von zeitraffendem Erzählen im narrativen Modus und zeitdeckendem Erzählen im dramatischen Modus, wobei bei ihm wegen der internen Fokalisierung Gedankenzitate dominieren.

Diese zwei Texte zeigen, dass das Thema Krankheit in der zeitgenössischen deutschsprachigen Literatur sehr aktuell ist und dass die Autoren vielfältige erzählerische Mittel für die Schilderung des Themas benutzen.

\section{Literaturverzeichnis}

\section{Primärliteratur}

Geiger, A. (2011). Der alte König in seinem Exil. München: Carl Hanser Verlag.

Ruge, E. (2011). In Zeiten des abnehmenden Lichts. Roman einer Familie (6. Aufl.). Reinbeck bei Hamburg: Rowohlt.

\section{Sekundärliteratur}

Aćimović, Lj. (2016). Интерна фокализација као наративна техника у роману "У временима замирућег свјетла" Ојгена Ругеа. Филолог - часопис за језик, књижевност и културу. (13), 268-286. doi: 10.21618/fil1613268a

Genette, G. (2010). Die Erzählung (3. Aufl.). Übersetzt von Adreas Knop, mit einem Nachwort von Jochen Vogt, überprüft und berichtigt von Isabel Kranz. Paderborn: Wilhelm Fink.

Hammelechle, S., \& Weyandt, H.-J.. Bestseller-Autor Arno Geiger: "Das Ende des Lebens ist auch Leben". Heruntergeladen von https://www.spiegel.de/kultur/literatur/bestseller-autor-arno-geiger-das-endedes-lebens-ist-auch-leben-a-745909.html. 5. Mai 2021.

Lämmert, E. (1955). Bauformen des Erzählens. Stuttgart: J. B. Metzlersche Verlagsbuchhandlung.

Martínez, M., \& Scheffel, M. (2016). Einführung in die Erzäh/theorie (10. überarb. und aktual. Aufl.). München: C. H. Beck.

Petersen, J. H. (1993). Erzählsysteme. Eine Poetik epischer Texte. Stuttgart: Verlag J. B. Metzler. 
Stanzel, F. K. (2001). Theorie des Erzählens (7. Aufl.). Göttingen: Vandenhoeck und Ruprecht.

Vogt, J. (1998). Aspekte erzählender Prosa. Eine Einführung in die Erzähltechnik und Romantheorie (8. Aufl.). Opladen: Westdeutscher Verlag.

\section{Internetquellen}

https://www.alzheimer-forschung.de/alzheimer/ 15. Mai 2021.

https://www.alzheimer-forschung.de/alzheimer/symptome/ 15. Mai 2021.

https://www.alzheimer-forschung.de/alzheimer/symptome/frueherkennung/ 15. Mai 2021.

https://de.wikipedia.org/wiki/In Zeiten des abnehmenden Lichts, 28. Mai 2021.

\section{Summary}

\section{NARRATIVE ANALYSIS OF THE PRESENTATION OF ILLNES IN THE OLD KING IN HIS EXILE AND IN TIME OF FADING LIGHT}

This paper analyzes the way two contemporary German writers problematize illness. The texts were published in 2011, both displaying autobiographical elements. In the autobiographical text The Old King in His Exile Arno Geiger writes about his father August who suffers from Alzheimer's disease, while all of his four children, his wife who lives separately and the caregiver who lives in their house take care of him. Dementia is just one of the illnesses affecting the members of Powileit/Umnitzer family from Eugen Ruge's novel In Times of Fading Light. The symptoms of dementia are different when it comes to Wilhelm Powileit and his stepson Kurt Umnitzer and can be compared with those from Geiger's text. Ruge's novel is also autobiographically motivated. The author himself is represented with the central character and the most significant reflector figure of the novel Alexander Umnitzer. The novel starts and ends in 2001: Alexander is diagnosed with reportedly terminal cancer, non-Hodgkin lymphoma, and goes on a trip to Mexico, leaving his severely demented father Kurt helpless at home. Alexander finds his inner peace in a small place in the Pacific, which will help him to reflect on his life, illness and death. The selected texts are analyzed according to the criteria of narratology at the level of discourse, whereby three concepts are explained and analyzed in the text: time, mood and voice. Finally, the conclusion problematizes the similarities and differences within the selected texts.

Key words: illness, Alzheimer, cancer, dementia, alcoholism 